\title{
Reverberation Mapping and Implications for Narrow-Line Seyfert 1 Galaxies
}

\author{
Bradley M. Peterson* \\ Space Telescope Science Institute and The Ohio State University \\ E-mail: peterson.12@osu.edu
}

\section{Elena Dalla Bontà}

Università di Padova and INAF - Padova

E-mail: elena.dallabonta@unipd.it

\begin{abstract}
Reverberation mapping is the principal method for determining the masses of the central black holes that power active galactic nuclei (AGNs). We discuss the importance of using line dispersion as opposed to full width at half maximum (FWHM) as the line-width measure in computing AGN black hole masses to avoid biasing (stretching) the AGN black hole mass scale. We argue that narrow-line Seyfert 1 (NLS1) galaxies are, for the most part, the low-luminosity extremes of a class of objects that should be defined by accretion rate rather than simply by line width.
\end{abstract}

Revisiting narrow-line Seyfert 1 galaxies and their place in the Universe - NLS1 Padova 9-13 April 2018

Padova Botanical Garden, Italy

${ }^{*}$ Speaker. 


\section{Introduction}

It is well established that masses of the supermassive black holes that power active galactic nuclei (AGNs) can be measured by reverberation mapping (RM). The simplest mass measurements are made by measuring the time delay $\tau$ between continuum and emission-line variations and combining this with the velocity width $\Delta V$ of the same emission line [14]. The mass is computed using the equation

$$
M_{\mathrm{BH}}=f\left(\frac{c \tau \Delta V^{2}}{G}\right),
$$

where $c$ is the speed of light and $G$ is the gravitational constant. Choice of the line-width measure will be discussed below, but in RM experiments it is common to use the line dispersion (second moment) of the emission line as measured in the rms residual spectrum in order to isolate the broad-line region gas that is actually responding to the continuum variations. The quantity in parentheses has units of mass and is often referred to as the "virial product." The factor $f$ subsumes everything we do not know, including and perhaps most importantly, the inclination of the system. The quantity $f$ can be determined directly by detailed modeling $[11,5]$; since most RM data are not suitable for detailed modeling, it is more common to use an average value, $\langle f\rangle$ which is useful if there is a secondary mass indicator that does not have to be especially accurate. It is common to use the well-known relation between black hole mass $M_{\mathrm{BH}}$ and host galaxy bulge velocity dispersion $\sigma_{*}[2,4,3]$ for this purpose. A recent determinatioon for $\mathrm{RM}$ AGNs is $\langle f\rangle \approx 4.47 \pm 1.24$ [22]. A more comprehensive overview of how RM is used to measure AGN black hole masses is provided by Peterson [13].

A key finding of RM is the well-defined relationship between the AGN luminosity $L_{\mathrm{AGN}}$ and the size of the BLR $R_{\mathrm{BLR}}=c \tau$ approximately of the form $R_{\mathrm{BLR}} \propto L_{\mathrm{AGN}}^{1 / 2}[6,1]$. This allows us to bypass the resource-intensive RM method and estimate $R_{\mathrm{BLR}}$ from the AGN luminosity alone: it is thus possible to estimate the black hole mass from a single spectrum (e.g., [8, 21, 9, 18, 19, 20, 7]).

\section{Computing Unbiased Masses}

As noted above, there are various ways to characterize the emission-line width $\Delta V$. The most common of these are full-width at half maximum (FWHM) and line dispersion

$$
\sigma_{\text {line }}=\frac{\int V_{\mathrm{LOS}}^{2} P\left(V_{\mathrm{LOS}}\right) d V_{\mathrm{LOS}}}{\int P\left(V_{\mathrm{LOS}}\right) d V_{\mathrm{LOS}}}
$$

where $P\left(V_{\mathrm{LOS}}\right)$ is the emission-line profile in line-of-sight (LOS) velocity; if the profile is Gaussian, this is just the standard deviation $\sigma$. Interestingly, a "typical" AGN emission-line profile is reasonably described by a Gaussian, which has the fixed ratio FWHM $/ \sigma_{\text {line }}=2.35$; unfortunately, this particular fact seems to have led to a pervasive belief that these two line-width measures are interchangeable and that one need only adjust the scaling parameter so that $f_{\mathrm{FWHM}}=f_{\sigma_{\text {line }}} /(2.35)^{2}$. The reason that this is incorrect is that the ratio FWHM $/ \sigma_{\text {line }}$ is a function of line width.

Which of these line-width measures give the more accurate mass determination? Peterson [12] attempted to address this issue at the last narrow-line Seyfert 1 (NLS1) meeting in Milan in 2011. We hoped at the time that repeated measurements of the masses of individual AGNs 
as they go through significant changes in lag and line width as the mean continuum level makes large excusions over time would eventually resolve this issue, but this approach is frustrasted by the fact that the individual mass measurements, as described above, have an intrinsic scatter of $\sim 0.4$ dex. At this level of accuracy, it matters little which line-width measure is used, particularly if the line profile is reasonably approximated by a Gaussian, The choice does become important, however, for extreme AGNs such as NLS1s and AGNs with double-peaked emission lines, where the emission-line profiles have large positive or large negative kurtosis. The strongest evidence that $\sigma_{\text {line }}$ is more accurate than FWHM actually comes from indirect mass measurements (i.e., using the $R_{\mathrm{BLR}}-L_{\mathrm{AGN}}$ scaling relationship to infer $R_{\mathrm{BLR}}$ from the luminosity) of large samples of QSOs. Consider specifically the case of QSO black hole masses determined from SDSS DR5 [16]. In this case, on account of the relatively low signal-to-noise ratio of the spectra, the lines were modeled with multiple Gaussians. The Gaussian models were then combined and the FWHM was measured from the model profile. Unfortunately, this leads to a bias in the distribution in the sense that use of FWHM leads to overestimation of the masses of quasars with broader lines and underestimation of the masses of quasars with narrower lines. This manifests itself as shown in the left panel of Figure 1; Steinhardt \& Elvis [17] identified a "sub-Eddington boundary" where higher-mass quasars fall further below the Eddington limit than lower-mass quasars. However, as shown in the right panel of Figure 1, Rafiee \& Hall [15] demonstrated that using the line dispersion as the line-width measure in the mass computation removed this effect.
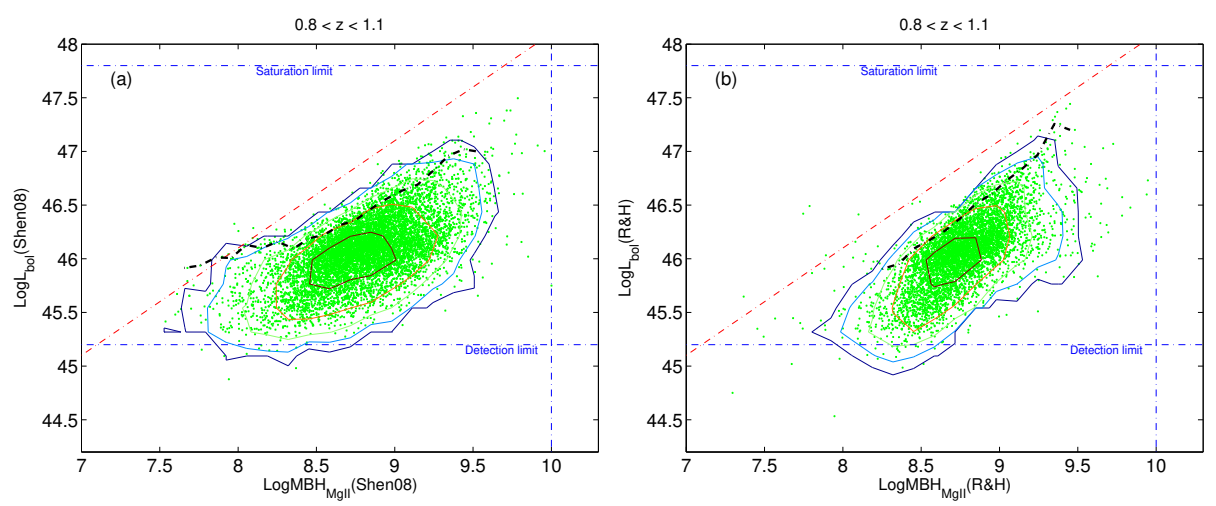

Figure 1: Left panel: Luminosity vs. black hole mass for DR5 QSOs in the redshift range $0.8<z<$ 1.1, with mass estimates based on FWHM as the line-width measure. The quasars with higher masses are artificially high and those at lower masses are artificially low because of the use of FWHM. This "rotates" the distribution in the clockwise direction so the higher mass quasars do not approach the Eddington limit Steinhardt \& Elvis [17] identify this as the "sub-Eddington boundary." Right panel: The same quasars are plotted as in the left panel, but now using the line dispersion $\sigma_{\text {line }}$ as the line-width measure to compute the masses. The upper envelope of the distribution is now roughly parallel to the Eddington limit, eliminating the artificial sub-Eddington boundary. From Rafiee \& Hall [15].

Unfortunately, FWHM is still often used as a line-width measure, primarily, we believe, because it's generally easy to measure and usually unambiguous. The line dispersion, on the other hand, is complicated by blending with other emission lines (e.g., $\mathrm{H} \beta$ is blended with both He II $\lambda 4686$ and broad Fe II complexes). Deblending emission features is generally not sufficiently 
straightforward that it can be done in a fully automated fashion, which mitigates against its use in large surveys, precisely where it is most needed. However, there is a clear relationship between FWHM and $\sigma_{\text {line }}$ that makes it possible to infer one from the other, with a small penalty due to intrinsic scatter in the relationship. By using measurements of $\mathrm{H} \beta$ line widths in the mean spectra of reverberation-mapped AGNs, we show in Figure 2 that the line dispersion can be inferred from FWHM using the best-fit relationship

$$
\log \sigma_{\text {line }}=3.276+0.495(\log \text { FWHM }-3.576) .
$$

One can thus transform FWHM to $\sigma_{\text {line }}$, but a penalty of 0.104 dex due to intrinsic scatter must be added in quadrature to the uncertainty in the line width and propagated to the mass uncertainty.

Figure 2: Line dispersion $\sigma_{\text {line }}$ as a function of FWHM for the broad $\mathrm{H} \beta$ emission line in the mean spectra of reverberation-mapped AGNs.

\section{Eddington Ratio or Inclination? The Reverberation Perspective}

The key question about NLS1s is whether the relatively small widths of the broad emission lines are due primarily to low inclination or high Eddington ratio, with most arguments coming down on the side of the latter. With new techniques for modeling reverberation spectra, it is possible to extract meaningful information about properties of the BLR beyond its mean size and velocity dispersion [10, 11, 5]. Here we revisit an argument made at the last NLS1 meeting in Milan [12]. Let us suppose that the physics of NLS1s is captured by Eddington ratio $\dot{m}=\dot{M} / \dot{M}_{\mathrm{Edd}} \propto \dot{M} / M_{\mathrm{BH}}$. Starting with the virial relationshiop (equation 1.1) and the radius-luminosity relationship $R_{\mathrm{BLR}} \propto$ $L_{\mathrm{AGN}}^{1 / 2}$, we can write the line width as

$$
\Delta V \propto\left(\frac{M_{\mathrm{BH}}}{L_{\mathrm{Edd}}^{1 / 2}}\right)^{1 / 2} \propto\left(\frac{M_{\mathrm{BH}}}{\dot{M}^{1 / 2}}\right)^{1 / 2} \propto\left(\frac{M_{\mathrm{BH}}}{\dot{m}}\right)^{1 / 4} .
$$

If the NLS1 phenomenon is defined by high values of $\dot{m}$, then the boundary between NLS1s and other AGNs should be defined by a line width that is a function of black hole mass. To minimize confusion, rather than attempting to redefine NLS1s, we will introduce a class of object we will call "high Eddington rate (HER)" sources as those with line widths

$$
\Delta V<2000\left(\frac{M_{\mathrm{BH}}}{10^{6.8} M_{\odot}}\right)^{1 / 4} \mathrm{~km} \mathrm{~s}^{-1}
$$

where the admittedly arbitrary normalization ensures the identify of most known NLS1s as HERs.

In Figure 3, we show model parameters for 9 AGNs for which dynamical models based on reverberation data have been obtained [11,5]. The filled circles represent HERs as defined by equation (3.1) and the open circles represent other AGNs. The second column confirms that the Eddington ratios of the HERs as defined from the simple mass estimates of equation (1.1) are indeed robust. Conversely, the fourth column does not show any dependence on BLR inclination, suggesting that the HER sources are defined by Eddington ratio, not inclination, as are the NLS1s. Given the small sample size, however, it difficult and dangerous to draw very strong conclusions. 


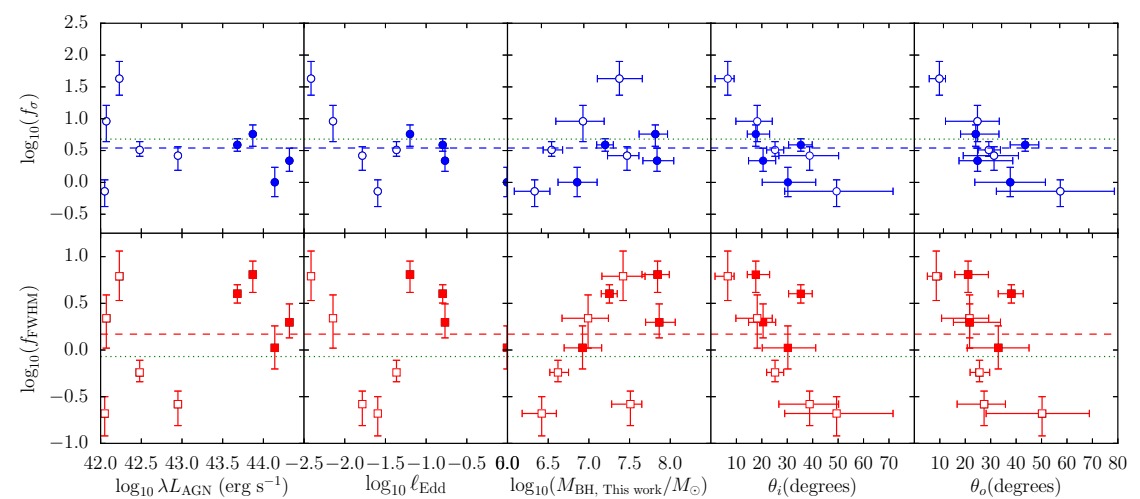

Figure 3: Model-derived properties for BLR models for the mass scaling factors ( $f$ in equation 1.1) for line dispersion (top row) and FWHM (bottom row). Left to right are AGN luminosity, Eddington ratio, black hole mass, BLR inclination, and torus opening angle. The filled circles are high Eddington ratio (HER) sources as defined here, and the open circles are other AGNs. From Grier et al. [5].

\section{Acknowledgements}

The authors are grateful to Pat Hall and Kate Grier for providing original copies of Figures 1 and 3, respectively. This conference has been organized with the support of the Department of Physics and Astronomy "Galileo Galilei", the University of Padova, the National Institute of Astrophysics INAF, the Padova Planetarium, and the RadioNet consortium. RadioNet has received funding from the European Union's Horizon 2020 research and innovation programme under grant agreement No 730562 .

\section{References}

[1] M.C. Bentz, K.D. Denney, C.J. Grier, et al. 2013, Ap.J., 767:149

[2] L. Ferrarese, \& D. Merritt 2000, Ap.J., 539, L9

[3] L. Ferrarese, R.W. Pogge, B.M. Peterson, D. Merritt, A. Wandel, \& C.L. Joseph 2001, Ap.J., 555, L79

[4] K. Gebhardt, R. Bender, G. Bower, A. Dressler, S.M. Faber, A.V. Filippenko, R.F. Green, C. Grillmair, L.C. Ho, J. Kormendy, T.R. Lauer, J. Magorrian, J. Pinkney, D. Richstone, \& S. Tremaine 2000, Ap.J., 539, L13

[5] C.J. Grier, A. Pancoast, A. Barth, et al. 2017, Ap.J., 849:186

[6] S. Kaspi, P.S. Smith, H. Netzer, D. Maoz, B.T. Jannuzi, \& U. Giveon 2000, Ap.J., 533, 631

[7] J.A. Kollmeier, C.A. Onken, C.S. Kochanek, A. Gould, D.H. Weinberg, M. Dietrich, R. Cool, A. Dey, D.J. Eisenstein, B.T. Jannuzi, E. Le Floc'h, \& D. Stern 2006, Ap.J., 648, 128

[8] A. Laor 1999, Ap.J., 505, L83

[9] R.J. McLure \& M.J. Jarvis 2004, MNRAS, 337, 109

[10] A. Pancoast, B.J. Brewer, \& T. Treu 2011, Ap.J., 730, 139

[11] A. Pancoast, B.J. Brewer, T. Treu, D. Park, A.J. Barth, M.C. Bentz, \& J.-H. Woo 2014, MNRAS, 445, 3073 
[12] B.M. Peterson 2011, in Proceedings of the Workshop "Narrow-Line Seyfert Galaxies and their Place in the Universe," POS (NLS1) 032

[13] B.M. Peterson 2014, Space Sci. Rev., 183, 253

[14] B.M. Peterson, L. Ferrerase, K.M. Gilbert, et al. 2004, Ap.J., 613, 682

[15] A. Rafiee \& P.B. Hall 2011, MNRAS, 415, 2932

[16] Y. Shen, J.E. Greene, M.A. Strauss, G.T. Richards, \& D.P. Schneider 2008, Ap.J., 680, 169

[17] C.L. Steinhardt \& M. Elvis 2010, MNRAS, 402, 2637

[18] M. Vestergaard 2002, Ap.J., 571, 733

[19] M. Vestergaard 2004, Ap.J., 601, 676

[20] M. Vestergaard \& B.M. Peterson 2006, Ap.J., 641, 689

[21] A. Wandel, B.M. Peterson, \& M.A. Malkan 1999, Ap.J., 576, 579

[22] J.-H. Woo, Y. Yoon, S. Park, \& S.C. Kim 2015, Ap.J., 801:38 